\title{
The Arabidopsis LYST INTERACTING PROTEIN 5 Acts in Regulating Abscisic Acid Signaling and Drought Response
}

\author{
Zongliang Xia ${ }^{1 *}$, Yongjin Huo ${ }^{1}$, Yangyang Wei', Qiansi Chen ${ }^{2}$, Ziwei Xu ${ }^{1}$ and Wei Zhang ${ }^{3}$ \\ ${ }^{1}$ College of Life Science, Henan Agricultural University, Zhengzhou, China, ${ }^{2}$ Zhengzhou Tobacco Research Institute of \\ CNTC, Zhengzhou, China, ${ }^{3}$ China National Tobacco Quality Supervision and Test Centre, Zhengzhou, China
}

Multivesicular bodies (MVBs) are unique endosomes containing vesicles in the lumens and play essential roles in many eukaryotic cellular processes. The Arabidopsis LYST INTERACTING PROTEIN 5 (LIP5), a positive regulator of MVB biogenesis, has critical roles in biotic and abiotic stress responses. However, whether the abscisic acid (ABA) signaling is involved in LIP5-mediated stress response is largely unknown. Here, we report that LIP5 functions in regulating ABA signaling and drought response in Arabidopsis. Analyses of a LIP5 promoter- $\beta$-glucuronidase (GUS) construct revealed

OPEN ACCESS

Edited by: Richard Sayre

New Mexico Consortium at Los Alamos National Labs, USA

Reviewed by:

J. C. Jang,

The Ohio State University, USA Michael Vincent Mickelbart, Purdue University, USA

${ }^{*}$ Correspondence: Zongliang Xia xiaz108@163.com

Specialty section: This article was submitted to Plant Physiology, a section of the journal Frontiers in Plant Science

Received: 17 September 2015 Accepted: 17 May 2016

Published: 01 June 2016

Citation:

Xia Z, Huo Y, Wei Y, Chen Q, Xu Z and Zhang W (2016) The Arabidopsis LYST INTERACTING PROTEIN 5 Acts in Regulating Abscisic Acid Signaling and Drought Response. Front. Plant Sci. 7:758. doi: 10.3389/fpls.2016.00758 substantial GUS activity in whole seedlings. The expression of LIP5 was induced by ABA and drought, and overexpression of LIP5 led to ABA hypersensitivity, enhanced stomatal closure, reduced water loss, and, therefore, increased drought tolerance. On the contrary, LIP5 knockdown mutants showed ABA-insensitive phenotypes and reduced drought tolerance; suggesting that LIP5 acts in regulating ABA response. Further analysis using a fluorescent dye revealed that $A B A$ and water stress induced cell endocytosis or vesicle trafficking in a largely LIP5-dependent manner. Furthermore, expression of several drought- or ABA-inducible marker genes was significantly downregulated in the lip5 mutant seedlings. Collectively, our data suggest that LIP5 positively regulates drought tolerance through ABA-mediated cell signaling.

Keywords: Arabidopsis, abscisic acid, drought, MVB biogenesis

\section{INTRODUCTION}

Adverse environmental conditions such as drought and high salinity have been becoming major limiting factors for plant growth and development. When encountering these stressful conditions, plants can respond and adapt to the adverse environment by triggering a network of signaling events to maintain cellular function (Hasegawa et al., 2000; Kawasaki et al., 2001; Zhu, 2002). Although, numerous drought-responsive genes have been characterized (Xiong et al., 2002; Zhu, 2002), the biological functions of many of these genes are essentially unknown in plants. Thus, it is desirable to understand the roles of these genes to improve crop adaptation to drought stress.

Abscisic acid (ABA) regulates many processes of plant growth and development, such as seed maturation, germination, and seedling growth (Leung and Giraudat, 1998; Finkelstein et al., 2002; Kermode, 2005; Christmann et al., 2006). Also, ABA is well-known for its regulatory roles in mediating adaptive responses to environmental stresses (Knight and Knight, 2001). For example, 
under drought stress, ABA induces stomatal closure to prevent water loss and launches a series of protective mechanisms to alleviate oxidative stress-induced cell damage in plants ( $\mathrm{Zhu}$, 2002; Yamaguchi-Shinozaki and Shinozaki, 2006).

In eukaryotic cells, multivesicular bodies (MVBs) are unique endosomes containing vesicles in the lumens. MVBs function as a protein degradation route in the endocytic pathway through which intraluminal vesicles can be delivered into and degraded upon fusion with lysosomes or vacuoles (Reyes et al., 2011; Contento and Bassham, 2012). In the MVB pathway, the ESCRT (endosomal sorting complexes required for transport) are dissociated or disassembled from the plasma membrane and recycled back into the cytoplasm (Reyes et al., 2011; Contento and Bassham, 2012). Hence, the MVB pathway has acted as a regulatory mechanism for removing damaged or downregulated proteins from the plasma membrane. The Vps4p/SKD1 AAA ATPase together with its positive regulator Vta1/LIP5 catalyzes the process of ESCRT disassembly in an ATP-dependent reaction (Babst et al., 1998; Fujita et al., 2004; Scott et al., 2005; Azmi et al., 2006; Lottridge et al., 2006; Winter and Hauser, 2006). Studies in both yeast and mammalian cells have indicated that both Vps4p/SKD1 and Vta1/LIP5 are critical players during MVB biogenesis (Yeo et al., 2003; Shiflett et al., 2004; Ward et al., 2005; Azmi et al., 2006). In metazoans, the ESCRT-III protein CHMP5 functions as a negative allosteric switch to control LIP5-mediated stimulation of VPS4 (Vild et al., 2015).

In higher plants, Arabidopsis LIP5 interacts with the AAA ATPase SKD1 and increases its ATPase activity by 4-5 folds in vitro (Haas et al., 2007). SKD1 participates in MVB function and contributes to vacuolar maintenance (Shahriari et al., 2010). Arabidopsis SKD1 knockout mutant is lethal and expression of the ATPase-deficient version SKD $1^{\mathrm{E} 232 \mathrm{Q}}$ also causes alterations in the endosomal system and ultimately cell death (Haas et al., 2007). Surprisingly, loss of function of LIP5 in Arabidopsis causes no phenotypic alterations under normal growth conditions, demonstrating that the activator LIP5 is dispensable for plant growth and development (Haas et al., 2007). Besides its function in MVB biogenesis, Arabidopsis LIP5 has been shown to play critical roles in biotic (resistance to Pseudomonas syringae; Wang et al., 2014) and abiotic stress tolerance (salt or heat stress; Wang et al., 2015). However, whether the ABA signaling is involved in LIP5-mediated stress response is largely unknown. In this study, we provide genetic evidence that LIP5 functions in regulating ABA signaling and drought response in Arabidopsis.

\section{MATERIALS AND METHODS}

\section{Plant Materials and Growth Conditions}

Arabidopsis (Arabidopsis thaliana ecotype Columbia [Col-0]) was used as the wild type in this study. Seeds of each genotype were surface sterilized with $10 \%$ bleach for $10 \mathrm{~min}$ and washed three times with sterile water. Sterilized seeds were then plated on Murashige and Skoog (MS) medium. Plants were stratified at $4^{\circ} \mathrm{C}$ in darkness for 3 days and then transferred to a growth chamber at $22^{\circ} \mathrm{C}$ with a 16 -h-light/8-h-dark photoperiod. After 1 week, seedlings were potted in soil and placed in a growth room at $22^{\circ} \mathrm{C}$, $60-70 \%$ relative humidity, a photoperiod of $16 \mathrm{~h} / 8 \mathrm{~h}$ (day/night) and light intensity of $150 \mu \mathrm{mol} \mathrm{m}^{-2} \mathrm{~s}^{-1}$, as described previously (Xia et al., 2014).

\section{Verification of LIP5 Arabidopsis Mutants}

The LIP5 T-DNA insertion mutants SALK_123717 (named lip5-s123) and SALK_145666 (named lip5-s145) seeds were obtained from the ABRC collection center (Ohio State University, Columbus). Homozygous mutants were identified by PCR from genomic DNA using forward primer P1, T-DNA left border primer LBb1, and LIP5 (accession number: At4g26750) genespecific reverse primer P2 (Supplementary Table S1), and analyzed further by DNA sequencing to confirm the insertions of the T-DNA in the gene. The transcript levels of LIP5 in the Col-0 and mutants were determined by quantitative RT-PCR.

\section{Real-Time PCR Analysis}

Real-time PCR was used to determine the expression pattern of LIP5 in different organs and in response to ABA treatment during seed germination and early seedlings growth. Total RNA extraction and first-strand cDNA synthesis were conducted as described previously (Xia et al., 2012). The qRT-PCR was performed in triplicate with an IQ5 light cycler system (Bio-Rad) using SYBR Premix ExTaq II (Takara, Japan) with gene-specific primers LIP5-QF and LIP5-QR (Supplementary Table S1), which produces a 157-bp product. The Arabidopsis Actin2 transcript was used as an internal control to quantify the relative transcript levels as described by us previously (Xia et al., 2014). The relative levels of transcripts were detected using the $2^{-\Delta \Delta C_{T}}$ method (Livak and Schmittgen, 2001).

To examine the relative expression of LIP5 in transgenic Arabidopsis plants, the expression of Actin2 was used as an internal control, and the wild-type was regarded as a standard and the relative level of gene expression was computed as described above. All qRT-PCR experiments were performed with three biological and three technical replicates.

\section{Construction of Plant Expression Vectors and Development of Transgenic Arabidopsis Lines}

For over-expression of LIP5, the LIP5 coding sequence was amplified and introduced into the binary vector pMW101 using primers LIP5-F with BamHI restriction site (underlined) and LIP5-R with XbaI restriction site (underlined; Supplementary Table S1), resulting in the transformation construct pMW10135S:LIP5.

For LIP5 promoter:GUS fusion construct, a DNA fragment covering 1,281 bp upstream of the translational start site of LIP5 was amplified by PCR using the primers LIP5P-F with EcoRI restriction site (underlined) and LIP5P-R with HindIII restriction site (underlined; Supplementary Table S1).This fragment was cloned into the EcoRI and HindIII sites of the binary vector pCAMBIA1381 containing a GUS reporter. GUS staining assay was done essentially as described previously (Bu et al., 2009). 
The binary constructs were introduced into Agrobacterium tumefaciens (strain GV3101) and then transformed into Arabidopsis wild-type (Col-0) or lip5 mutants via the floral dip method (Clough and Bent, 1998). Transgenic lines were selected by germinating seeds on MS medium containing $50 \mathrm{mg} / \mathrm{L}$ hygromycin. After 2 weeks on selection medium, green seedlings (T1 plants) were transferred to soil and grown to maturity to set seeds (T2 seeds) in a growth room. T2 seeds were germinated on antibiotics-selective medium again and the one-copy lines were identified by examining the segregation ratio $(3: 1)$ of the antibiotics-selectable marker. Each one-copy line was maintained growth to set seeds until $\mathrm{T}_{3}$ generation. Homozygous T3 lines were used for ABA response or GUS staining assays.

\section{In silico Analysis of LIP5 Promoter}

To identify putative cis-acting regulatory elements in the promoter region of LIP5, the online analysis tool PlantCARE ${ }^{1}$ was used.

\section{Root Growth of Seedlings}

To study the effect of ABA on root growth of seedlings, seeds were sown on ABA-free medium as described above. $24 \mathrm{~h}$ after stratification, germinated seeds were transferred to medium containing different concentrations $(0,1$, or $2 \mu \mathrm{M})$ of ABA. Plates were placed vertically in a growth chamber, and root growth was measured at 5 days after the end of stratification.

\section{Drought Treatment and Leaf Water Content Measurement}

For gene expression analysis, 2-weeks-old seedlings from the agar plate were transferred onto a filter paper in a covered Petri dish and subjected to dehydration treatment. The treatment was conducted in an environment of $70 \%$ relative humidity. For the drought tolerance test on the soil-grown plants, 1week-old seedlings were transplanted to the soil for additional 2 weeks under standard growth conditions, and then plants were subjected to progressive drought by withholding water for 12 days and then re-watered for 4 days. Survival rates (\%) under drought treatment were determined as the number of visibly green plants after 4 days. To minimize experimental variations, the same number of plants was grown on the same tray. After 8 days without watering, weight of leaf samples from transgenic, mutant and wild-type plants under stressed and control conditions was determined as fresh weight (FW), and then the samples were dried at $80^{\circ} \mathrm{C}$ for $24 \mathrm{~h}$ and weighed as dry weight (DW). The water content was calculated as (FW$\mathrm{DW}) / \mathrm{FW} \times 100 \%$. The entire test was conducted at least three times.

\section{Stomatal Aperture Bioassay}

Leaves from 3-weeks-old plants grown under the same conditions were harvested in darkness. Epidermal peels were stripped and incubated in a solution $\left(50 \mathrm{mM} \mathrm{KCl}, 10 \mathrm{mM} \mathrm{CaCl}_{2}\right.$, and

\footnotetext{
${ }^{1}$ http://bioinformatics.psb.ugent.be/webtools/plantcare/html/
}

$10 \mathrm{mM}$ MES-KOH, $\mathrm{pH}$ 6.15), and exposed to light for $1.5 \mathrm{~h}$. Subsequently, $50 \mu \mathrm{M}$ ABA was added to the solution to assay for stomatal closing. After treatment for $3 \mathrm{~h}$, stomatal apertures were measured as described (Xia et al., 2013) with a microscope. The apertures of usually 30-40 stomata were measured in two independent experiments.

\section{GUS Bioassays}

Seeds and young seedlings at different developmental stages, and different parts from mature transgenic plants, were collected and used for histochemical detection of GUS expression (Jefferson et al., 1987). Materials were stained at $37^{\circ} \mathrm{C}$ overnight in the solution [1 mg/mL 5-bromo-4-chloro-3-indolyl- $\beta$-D-glucuronic acid (X-Gluc), $5 \mathrm{mM}$ potassium ferricyanide, $5 \mathrm{mM}$ potassium ferrocyanide, $0.03 \%$ Triton $\mathrm{X}-100$, and $0.1 \mathrm{M}$ sodium phosphate buffer, $\mathrm{pH}$ 7.0], and then were photographed after GUS staining.

\section{Fluorescence Microscopy}

Fluorescent microscopy analysis of a styryl dye FM1-43 (SigmaAldrich) internalization in Arabidopsis root or leaf guard cells was conducted as described previously (Wang et al., 2015). For ABA-induced vesicle trafficking observation, the detached leaves from 2 weeks-old plants with $1 \mathrm{~h}$-ABA $(50 \mu \mathrm{M})$ treatment were used. For drought-induced vesicle trafficking observation, the excised roots from 10 days-old seedlings with $30 \mathrm{~min}$ PEG 6000 (10\%) treatment were collected. Leaf epidermal peels or root samples were stained in $10 \mu \mathrm{M}$ of FM1-43 for $20 \mathrm{~min}$ at room temperature, washed and examined under a confocal microscope. For confocal laser microscope scanning, the wavelength of the confocal microscopy was set to be $488 \mathrm{~nm}$ excitation and 600-650 $\mathrm{nm}$ emission. About $8 \sim 10$ independent leaves or roots were quantified and statistically analyzed for each genotype/treatment.

\section{Accession Numbers}

Sequence data from this article can be found in the GenBank/EMBL data libraries under the following accession numbers: LIP5, At4g26750; RAB18, At5g66400; RD29A, At5g52310; RD29B, At5g52300; KIN1, At5g15960; RD22, At5g25610; ADH1, Atlg77120.

\section{Statistical Analysis}

The data were presented as the mean \pm standard error (SE) of three independent experiments. Statistical analyses were conducted using the data processing software SPSS V.16.0 (SPSS, Inc., USA). For all analyses, the significant level was set at $P<0.05$.

\section{RESULTS}

\section{Expression Pattern of LIP5}

The transcriptional pattern of LIP5 was examined in young seedlings and in multiple organs of more mature plants by qRT-PCR. As shown in Figure 1A, the transcripts of LIP5 were detected in young seedlings, roots, stems, leaves, and flowers of 
A

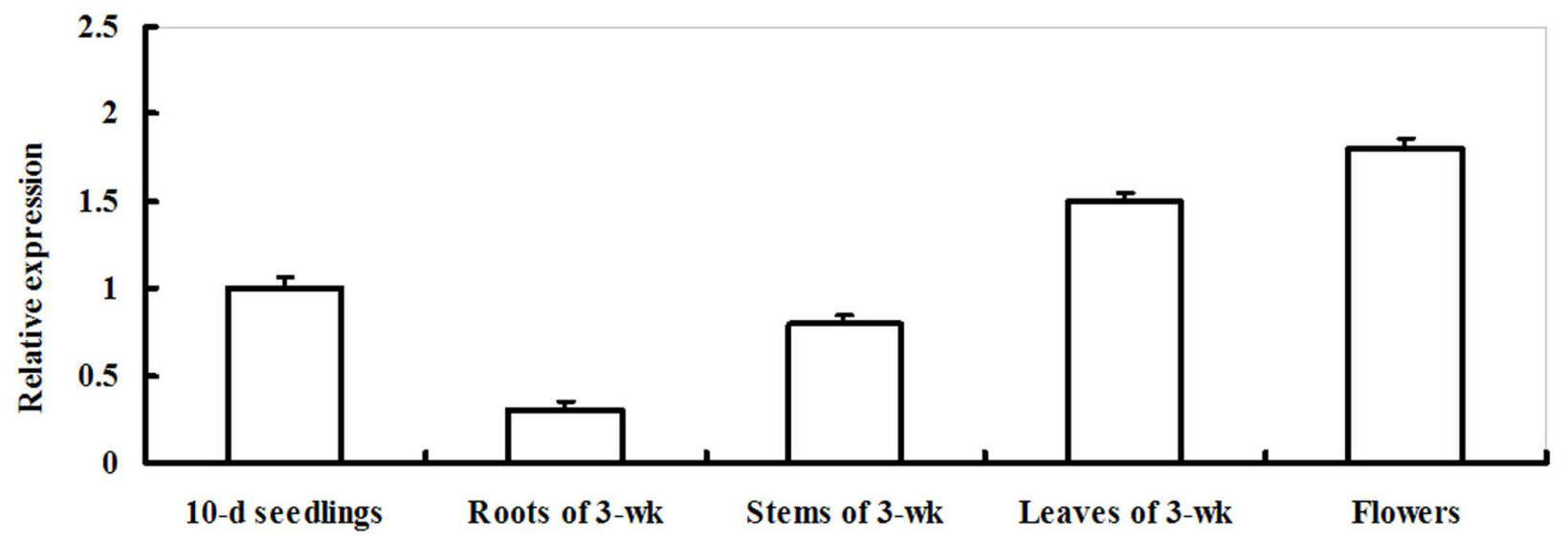

B
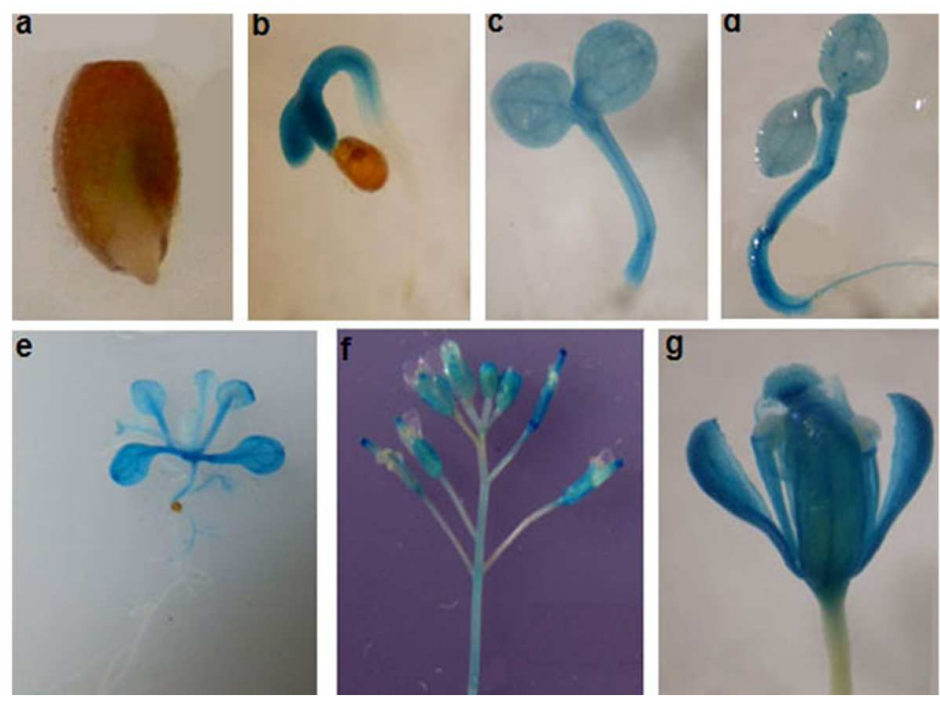

FIGURE 1 | Expression patterns of LIP5. (A) qRT-PCR analysis of LIP5 expression in different organs. Transcript levels of LIP5 were quantified by qRT-PCR against Actin2. For each assay, the expression level at 10-days-old seedlings stage was defined as 1.0 and three technical replicates were conducted. Data shown are mean \pm SE of three independent experiments. (B) GUS staining of LIP5pro:GUS transgenic plants from different growth stages. (a) 1-day-old germinating seedling; (b) 2-days-old seedling; (c) 3-days-old seedling; (d) 4-days-old seedling; (e) 2-weeks-old seedling; (f) inflorescences from 7-weeks-old plants; (g) flower from 7 -weeks-old plants. Experiments were repeated at least two times with similar results.

more mature plants. The LIP5 transcript levels were significantly high in leaves and flowers. In contrast, LIP5 transcripts were low in roots (Figure 1A).

For a more detailed analysis of the LIP5 expression pattern, a 1,281-bp promoter sequence of LIP5 was fused with the GUS gene to generate transgenic plants LIP5pro:GUS and to follow LIP5 expression in different developmental stages. Histochemical staining showed GUS activity at all developmental stages tested, from seed germination to flowering (Figure 1B). This indicates that LIP5 is expressed at all developmental stages and throughout the Arabidopsis plant. GUS expression was first detected in 1-dayold germinated seeds with weaker GUS staining in the emerging radicle (Figure 1Ba). Higher GUS expression was detected in hypocotyls of 2- and 3-days-old seedlings (Figures $\mathbf{1 B b}, \mathbf{c}$ ). At 4 days, GUS expression was detected throughout the plant (Figure 1Bd). In 2- or 7-weeks-old plants, GUS staining was clearly observed in leaves or inflorescences, but relatively weak in roots (Figures $1 \mathrm{Be}-\mathrm{g}$ ).

\section{Induction of the LIP5 Gene in Arabidopsis Leaves by ABA and Drought Stress}

Time-course analysis of LIP5 transcript levels in Arabidopsis plants under ABA or dehydration stress was performed by qRTPCR (Figures 2A,B). The transcript levels of LIP5 were increased rapidly after $0.5 \mathrm{~h}$, and then remained higher levels during 

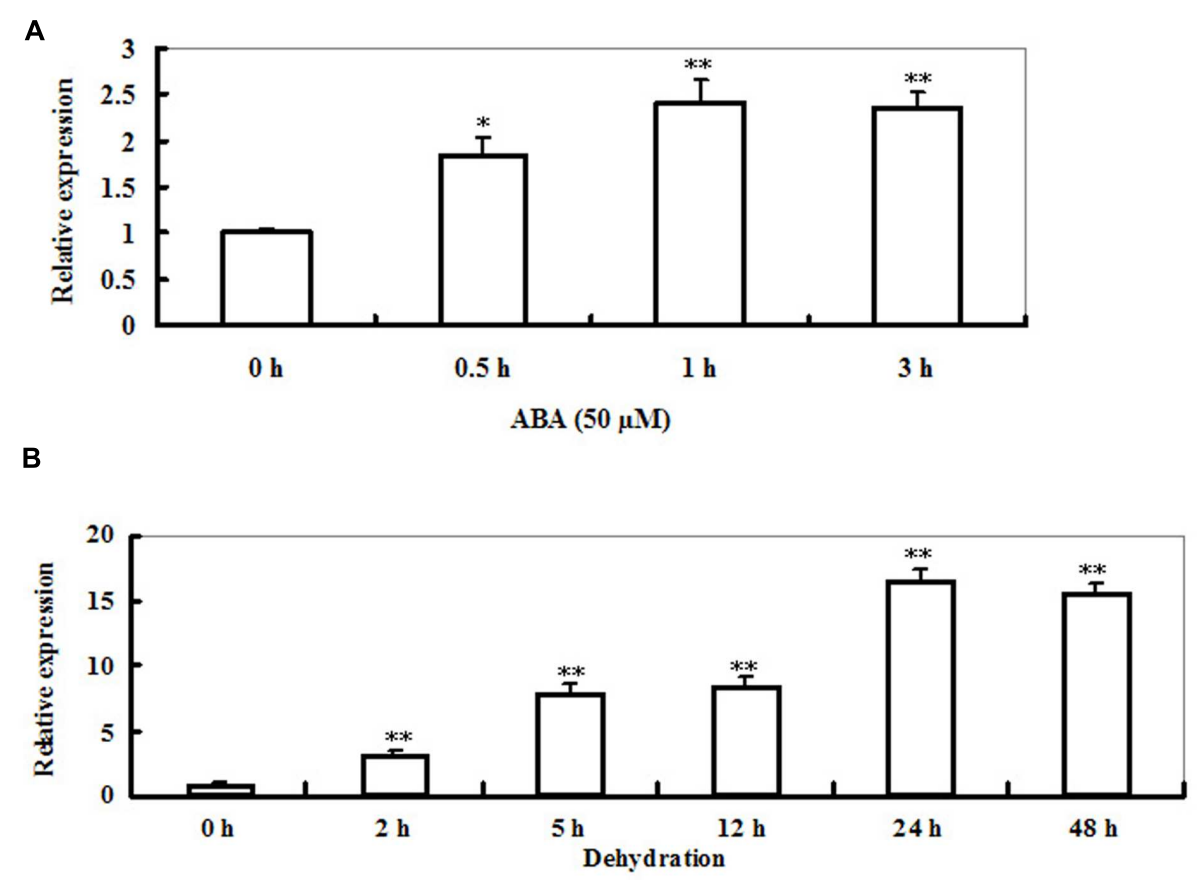

FIGURE 2 | Expression of LIP5 in response to abscisic acid (ABA) or dehydration. (A) ABA-induced LIP5 expression revealed by qRT-PCR. Two-weeks-old wild-type seedlings were treated with $50 \mu \mathrm{M}$ ABA for $0,0.5,1$, and $3 \mathrm{~h}$. Actin2 was used as an internal control. (B) Dehydration-induced LIP5 expression revealed by qRT-PCR. Two-weeks-old wild-type seedlings were placed on Whatman paper and then harvested at 0, 2, 5, 12, 24, and 48 h. In both (A,B) Actin2 was used as an internal control. For each experiment, three technical replicates were conducted. Data shown are mean $\pm \mathrm{SE}$ of three independent experiments. $T$-test, with ${ }^{* *} P<0.01 ;$-test, with ${ }^{*} P<0.05$.

$3 \mathrm{~h}$ period of ABA treatment with a peak at $1 \mathrm{~h}$ (about 1.5fold increase in transcripts; Figure 2A). Accumulation of LIP5 transcripts was first detected $2 \mathrm{~h}$ after the dehydration treatment and reached a maximal level at $24 \mathrm{~h}$ during $48 \mathrm{~h}$ period of the stress (Figure 2B). The LIP5 transcripts were upregulated by 16fold after $24 \mathrm{~h}$ of exposure to dehydration. These data suggest that LIP5 could be involved in the responses to ABA or drought stress.

\section{Genetic Complementation of the Arabidopsis lip5 Knockdown Mutants in Response to ABA}

To explore the LIP5 function in response to ABA treatment, two independent homozygous T-DNA insertion lines SALK_123717 (named lip5-s123) and SALK_145666 (named lip5-s145) were identified from the ABRC seed stock center. Analysis of the fulllength cDNA and the genomic sequences revealed that LIP5 is composed of six exons and five introns (Supplementary Figure S1A). The gene is located on chromosome IV of the Arabidopsis genome. In both lines, the T-DNA was inserted in the region of $300 \mathrm{bp}$ upstream of the translational start site of LIP5. The T-DNA insertion positions are illustrated in Supplementary Figure S1A and homozygous mutants were verified using LIP5 gene-specific and T-DNA border primers by PCR analysis (Supplementary Figure S1B). In both homozygous lines, a decrease of $60 \%$ approximately in abundance of LIP5 mRNA was detected by qRT-PCR compared with the same aged wild-type plants (Figure 3A). Both T-DNA insertion lines had comparable levels in LIP5 transcripts, so the lip5-s123 line was chosen for further analysis.

The lip5 mutant (lip5-s123), lip5-s123/35S-LIP5 line (lip5comp), along with wild-type (Col-0) were also assessed for their responses to $\mathrm{ABA}$ by investigating the retardation of seedling root growth. Under 1 or $2 \mu \mathrm{M}$ of $\mathrm{ABA}$, root growth of both wild-type and the lip5-comp plants was more severely inhibited than that of the lip5 mutant (Figures 3B,C). Noticeably, the lip5comp line is actually an over-expression line (lip5-s123/35S-LIP5), but it behaved like the wild-type plants in the ABA assays. To clarify these results, we detected the transcript levels of LIP5 between wild type and the lip5-comp line used in the ABA assays using qRT-PCR. As a result, there were no significant differences in the transcript levels of LIP5 between wild type and the lip5-comp line, which could explain the observation (Data not shown). These results demonstrated that lip5 mutant was less sensitive to ABA than the wild type; moreover, LIP5 effectively rescued the ABA-insensitive phenotypes of the lip5 knockdown mutants.

\section{ABA Response of LIP5 Mutant and OE Lines}

To further investigate the function of LIP5 in response to ABA, wild-type Arabidopsis plants overexpressing the LIP5, 


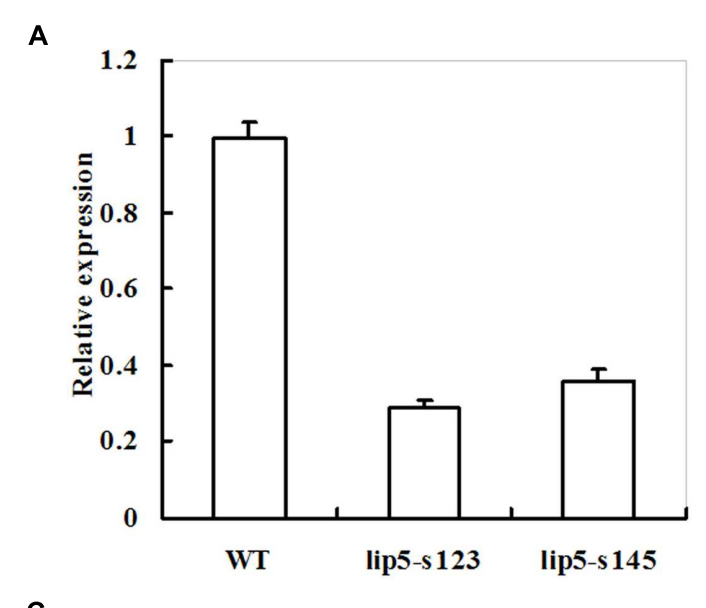

B

C
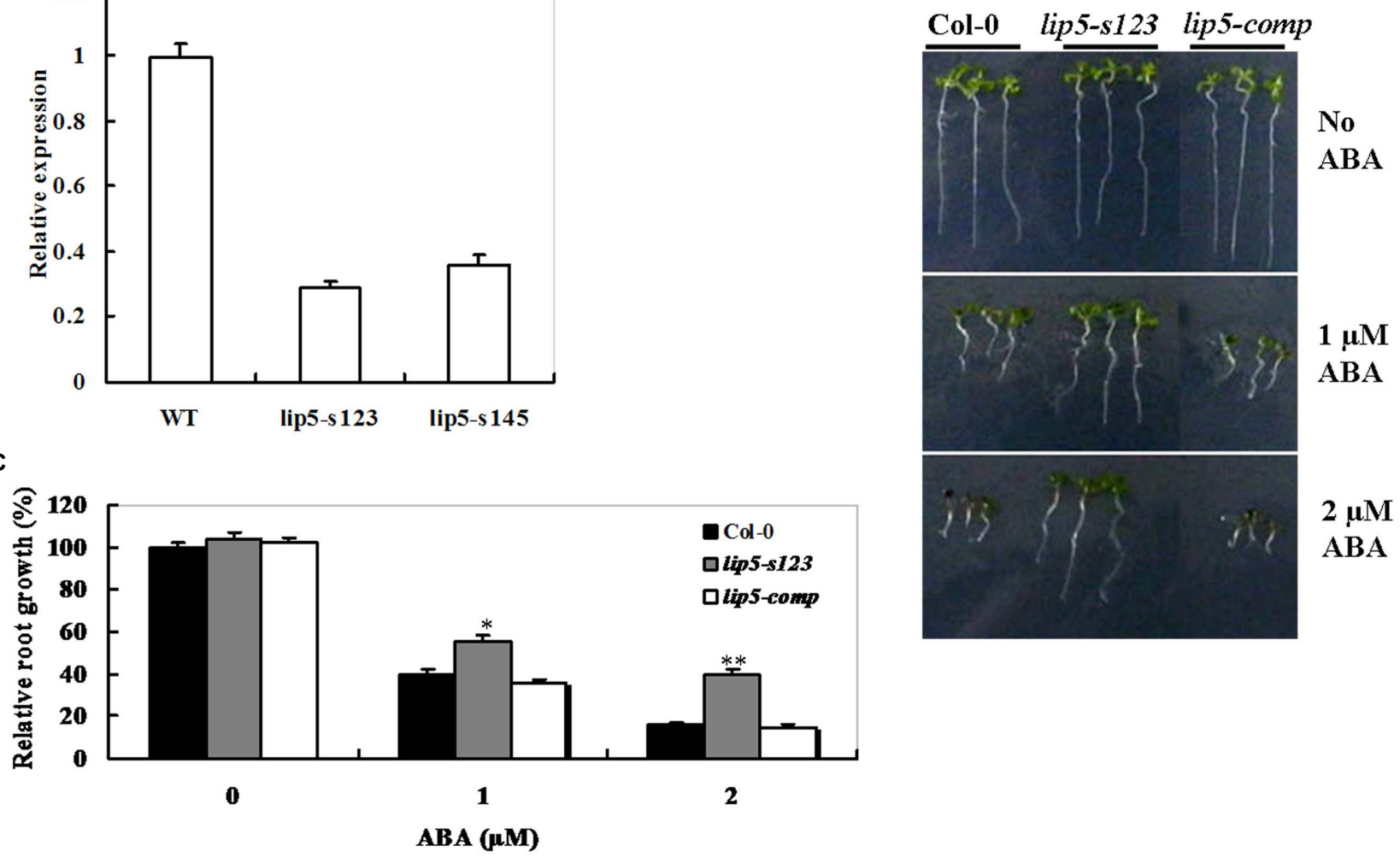

FIGURE 3 | Genetic complementation of the lip5 mutant in response to ABA. (A) Transcript levels of AtLIP5 in the Col-0 and lip5-s123 and lip5-s145 mutants determined by RT-PCR. (B) Representative phenotypes of seedlings at 7 days after the end of stratification. Twenty-four hours after stratification, germinated seeds were transferred from ABA-free medium to medium containing 1 or $2 \mu \mathrm{M}$ ABA. (C) Root growth measurements. Seedling root length was measured at 7 days after the end of stratification. Relative root growth compared with that on ABA-free medium is indicated. Data show the mean $\pm \mathrm{SE}$ of three replicates. At least 30 seedlings per genotype were measured in each replicate. $T$-test, with $* * P<0.01$; $t$-test, with $* P<0.05$. In both (B,C) experiments were repeated at least two times with similar results.

under the control of the $35 \mathrm{~S}$ promoter, were generated. Five homozygous lines (T3 generation) were obtained, and two lines (WT/35S-LIP5\#3 and \#9) exhibiting high levels of transgene expression (Figure 4A) were selected for phenotypic characterization. The LIP5 mutant (lip5-s123) and LIP5 OE lines (WT/35S-LIP5\#3 and \#9) were also examined for their $\mathrm{ABA}$ response by investigating the retardation of seedling root growth. When grown on the medium with 1 or $2 \mu \mathrm{M}$ of $\mathrm{ABA}$, root growth inhibition of lip5-s123 seedlings was less than that of the wild type, whereas both OE lines were more severely inhibited (Figure 4B). Relative root length determination also showed significant differences among the mutant, OE lines and wild-type plants under 1 or $2 \mu \mathrm{M}$ of ABA (Figure 4C). These indicate that lip5-s123 is less sensitive to ABA but OE lines are hypersensitive. Taken together, the contrasting ABA sensitivities between lip5 mutant and LIP5 OE lines suggest that LIP5 may act as a positive regulator of ABA signaling during seedling development.

\section{LIP5 Affects Drought Tolerance of Plants}

To test the role of LIP5 in regulating drought response of plants, 3-weeks-old 35S:LIP5, lip5 mutant, and wild-type plants were used for drought tolerance assays in soil. As shown in Figure 5A, after 12 days without watering, most of the 35S:LIP5 plants survived the water stress and upper leaves of these plants were still green and fully expanded, whereas most of lip5 and wild-type plants displayed severe wilting (especially for the lip5 plants, all leaves were severely curled and partial leaves were turning yellow). After re-watering, 35S:LIP5 plants showed a high survival rate (more than 90\%), whereas the corresponding survival rates were 39\% for wild-type plants and 19\% for lip5 mutants (Figure 5A); indicating that LIP5 plays a positive role in drought tolerance. Consistent with their drought-tolerant performance, the water content of both OE lines after 8 days without watering was significantly higher than that of the wildtype and lip5 mutant plants (Figure 5B); suggesting that the improved drought tolerance of both OE lines resulted from decreased water loss from plants.

Several studies have established that high sensitivity to ABA leads to increased drought tolerance by stomatal aperture measurement (Lim and Lee, 2014). To determine whether the differential drought-tolerant phenotypes were associated with their enhanced ABA sensitivity, we measured ABA-mediated stomatal aperture (Figure $5 \mathrm{C}$ ). In the absence of $\mathrm{ABA}$, there 


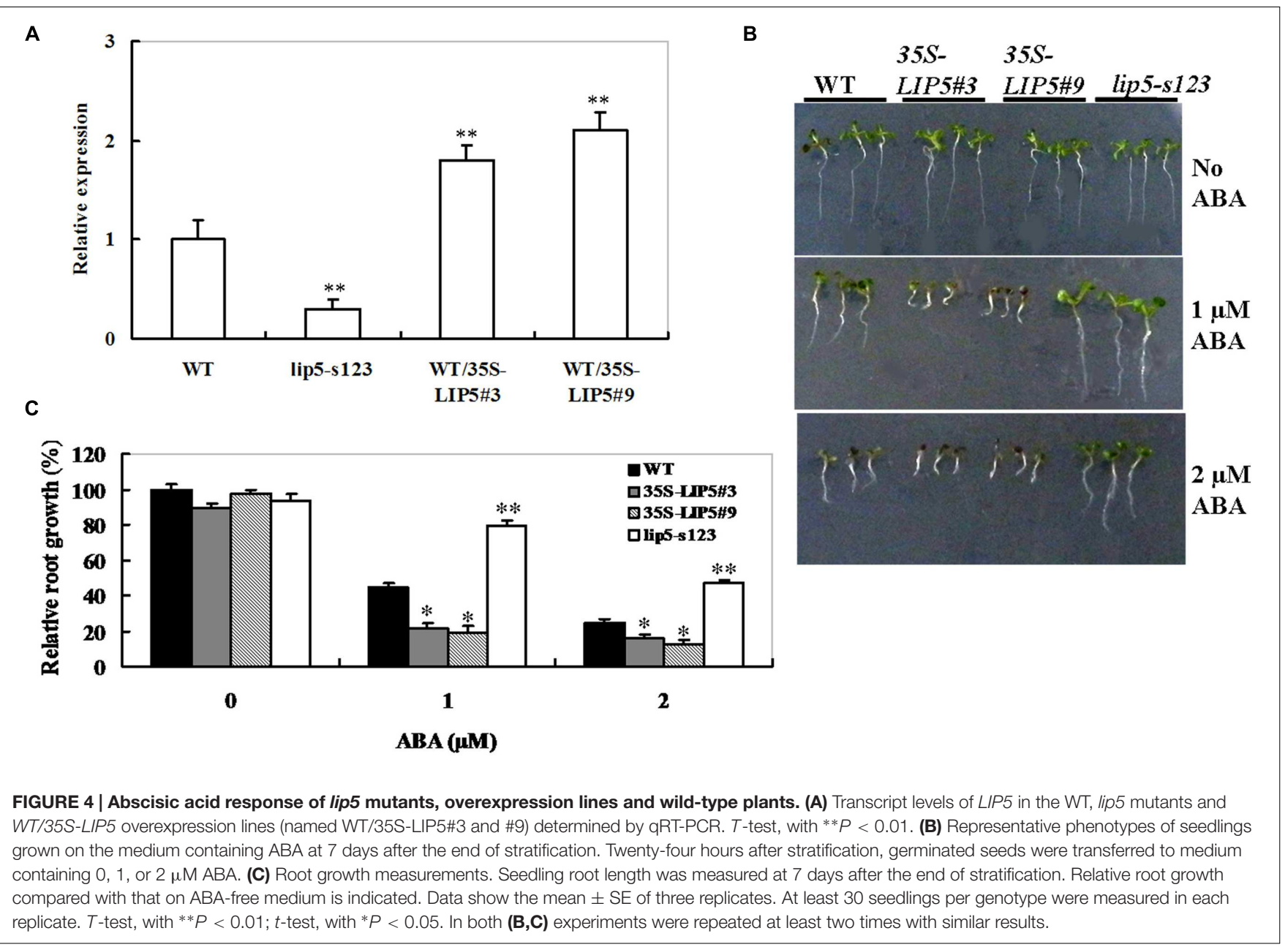

was no obvious difference in stomatal aperture among wild-type, lip5 mutant and OE plants. However, the stomatal pore sizes were significantly reduced in both $\mathrm{OE}$ plants compared with wild-type plants after ABA treatment. The stomatal apertures of wild-type and OE plants were reduced by 40 and 58-60\%, respectively; whereas those of lip5 plants were only reduced by 26\% (Figure 5C); demonstrating that LIP5 OE plants were more sensitive than the wild-type in ABA-induced stomatal closure, whereas the lip5 mutant plants were less sensitive. These data indicate that LIP5 may act in regulating ABA-mediated stomatal closure.

\section{Cell Endocytosis or Vesicle Trafficking Induced by Stress- or ABA in LIP5 Mutant and Wild Type Plants}

It has been recently shown that LIP5 exerts its regulatory roles in pathogen- and salt-induced cellular vesicle trafficking (Wang et al., 2014, 2015). To examine whether different levels of tolerance between the mutant and wild type plants were also associated with vesicle trafficking under stress conditions, we compared WT and lip5 mutant plants for drought-induced endocytic activities using FM1-43 as a fluorescent endocytosis marker. The membrane-selective FM1-43, whose fluorescent signals are located in a lipid-rich membrane, can enter the cells by endocytic vesicles derived from plasma membrane (Bolte et al., 2004). Without PEG (on MS medium), FM1-43 fluorescent signals were predominantly located at the plasma membrane as a result of the association of the dye with the lipid phase (Figure 6A, left lane). Moreover, no significant difference was observed in internalized FM1-43 signals between WT and lip5 mutant root cells (Figures 6A,B). After 30 min-PEG (10\%) treatment, the fluorescent signals at the plasma membrane were reduced and became diffusive and strong in the cytoplasm. This was clearly seen in the root epidermal cells of the wild type (Figure 6A, right lane). The intensity of internalized FM1-43 signals increased by almost 1.5 folds in WT roots (Figure 6B). Unlike WT plants, however, there was only 50\% increase in the fluorescence signals in the lip5 roots (Figure 6B). Also, ABA-induced vesicle trafficking in guard cells of leaf epidermis was observed. Without ABA treatment, the fluorescent signals were evenly associated with the plasma membrane in both wild-type and lip5 guard cells (Supplementary Figure S2A). Moreover, there was no significant difference in internalized fluorescent signals between wild-type and lip5 (Supplementary Figure S2A). Surprisingly, after $1 \mathrm{~h}$-ABA treatment, the 


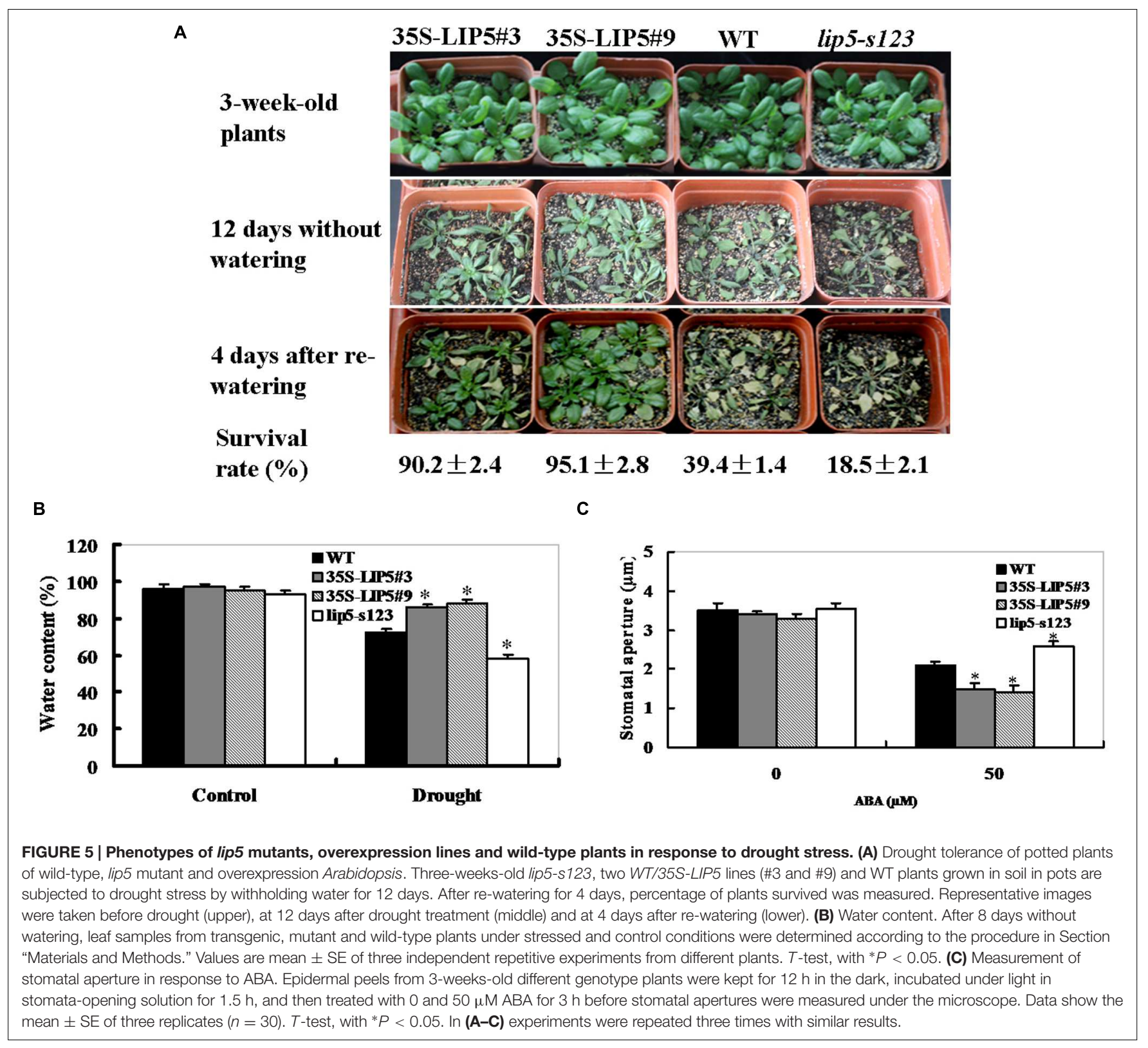

internalized fluorescent signals were increased at the plasma membrane and became intense. Moreover, the ABA-induced stomatal closure was clearer in the wild type than the mutant (Supplementary Figure S2A). The internalized FM1-43 signals increased by $180 \%$ in the wild type, but only $85 \%$ in the lip5 (Supplementary Figure S2B). These results indicate that drought or ABA induces cell endocytosis or vesicle trafficking in a largely LIP5-dependent way.

\section{LIP5 Affects Expression of Drought- or ABA-Responsive Genes}

Generally, drought sensitivity is correlated to transcripts levels of drought- or ABA-related genes (Xiong et al., 2002; Xia et al., 2013). The expression levels of LIP5 are positively correlated with both ABA sensitivity and drought tolerance, so we next wondered whether LIP5 affected the expression profiles of ABA-induced or drought stress-related genes. Both LIP5 OE lines and lip5 mutant together with wild-type seedlings were treated or mock treated with $\mathrm{ABA}$ to determine the expression of several drought- or ABA-inducible marker genes. qRT-PCR assays indicated that ABA treatment resulted in increased expression of several ABA-inducible marker genes, including $R A B 18$ (Responsive to $A B A$ 18), RD22 (Responsive to dehydration 22), RD29A, RD29B, ADH1 (alcohol dehydrogenase 1) and KIN1 (Kurkela and Borg-Franck, 1992; Lang and Palva, 1992; Abe et al., 2003) in wild-type seedlings (Figure 7). The ABA-induced expression of these marker genes was generally intensified in both LIP5 OE lines (increased averagely by $0.6 \sim 1.7$-fold at $3 \mathrm{~h}$ compared with the wild-type), but 
A
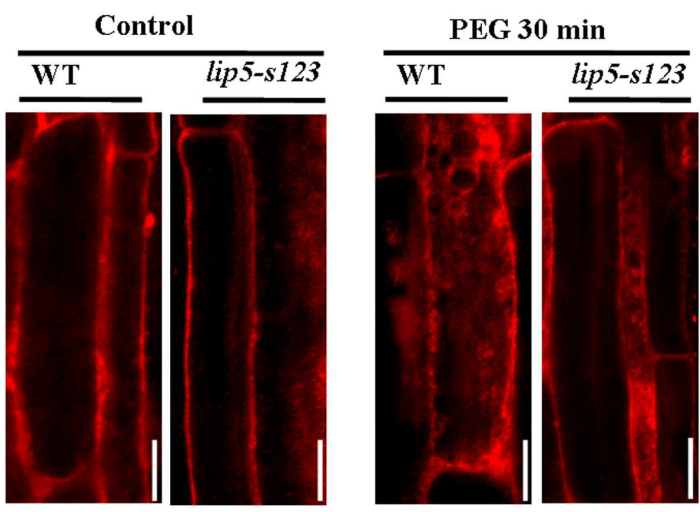

B

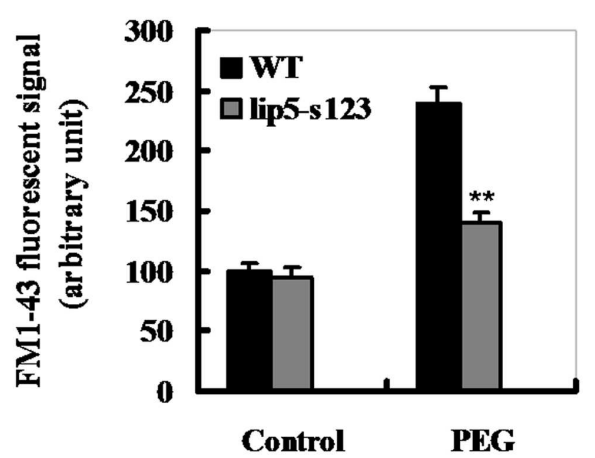

FIGURE 6 | Phenotypes of LIP5 mutant and wild type plants in drought-induced endocytosis. Ten days-old lip5-s123 and WT seedlings on MS medium were carefully transferred to MS plates containing 0 or $10 \%$ of PEG 6000 for $30 \mathrm{~min}$. Drought-induced endocytosis was analyzed using FM1-43 staining. (A) Representative confocal images of Arabidopsis root epidermal cells of WT and lip5 mutant plants after FM1-43 staining. Bar $=50 \mu \mathrm{m}$. (B) Signal intensity of internalized FM1-43 in Arabidopsis root epidermal cells of WT and lip5 mutant plants. Means and SE were calculated from images of 8 independent roots (10 images/root) for each genotype. $T$-test, with ${ }^{* *} P<0.01$. In both $(\mathbf{A}, \mathbf{B})$ experiments were repeated two times with similar results.

significantly attenuated in lip5 seedlings (decreased by $0.4 \sim 0.6$ fold at $3 \mathrm{~h}$ compared with the wild-type; Figures 7A-F). These results indicate that the LIP5 may function as a positive regulator in ABA-mediated drought tolerance by upregulating expression of some $\mathrm{ABA}$ - or drought-responsive genes.

\section{DISCUSSION}

The MVB pathway plays essential roles in several eukaryotic cellular processes. Proper function of the MVB pathway requires reversible membrane association of the ESCRTs, a process catalyzed by SKD1 AAA ATPase. LIP5, a plant homolog of Vps twenty associated 1 (Vta1), is a positive regulator of MVB biogenesis (Haas et al., 2007). Moreover, LIP5 plays a critical role in pathogen/stress-responsive MVB biogenesis in plant stress responses (Wang et al., 2014, 2015). However, little is known about the relationship between the ABA signaling and LIP5-mediated stress responses. We report here LIP5 plays a positive role in regulating ABA signaling and drought response. Overexpression of LIP5 leads to $\mathrm{ABA}$-associated phenotypes such as $\mathrm{ABA}$ hypersensitivity in seedling growth (Figures $\mathbf{4 B}, \mathbf{C}$ ), enhanced stomatal closure (Figure 5C), reduced water loss (Figure 5B), and, therefore, increased drought tolerance (Figure 5A). On the contrary, the lip5 mutant showed ABA-insensitive phenotypes and reduced drought tolerance (Figures 3-5). These data indicate that LIP5 may be a positive regulator in the ABA signal transduction pathway.

We considered the possibility that LIP5 positively participated in ABA signaling regulation in response to drought stress in Arabidopsis based on the following reasons. First, LIP5 was rapidly induced by ABA and water stress (Figures 2A,B). Second, the lip5 knockdown mutants and LIP5-overexpressing transgenic plants exhibited opposite sensitivities to ABA in seedling growth (Figures 4B,C). Third, LIP5 positively regulated ABA-induced stomatal closure, which may reduce transpiration water loss in response to drought stress (Figures 5B,C). Phenotypic analysis indeed demonstrated that LIP5-overexpressing transgenic plants were highly tolerant to drought stress, as they survived without watering for 12 days (Figure 5A). In contrast, the lip5 mutant line was susceptible to the stress and lost leaf water faster than wild-type plants (Figures 5A,B). Finally, in silico analysis of LIP5 promoter region (1, 281-bp upstream of LIP5 ORF) with the PlantCARE program (Lescot et al., 2002) identified several consensus cis-acting elements related to stress responses such as ABRE (ABA-responsive element), TC-rich repeats and TCA-element (Supplementary Table S2). These cis-acting elements are involved in responses to ABA, drought or stress defense in Arabidopsis (Nakashima et al., 2006). Noticeably, both T-DNA insertion mutants, in which the T-DNA was inserted into the region close to the second ABRE motif in the promoter of the LIP5 gene, showed reduced ABA sensitivity in our study. Overall, these results led us to propose that LIP5 may be a positive regulator of an ABA-dependent response to drought stress in Arabidopsis.

LIP5 is a positive regulator, but it is not an essential component of MVB biogenesis. Wang et al. (2014, 2015) demonstrated that the hypersensitivity of lip 5 knockout mutants to pathogen infection, heat or salt stress is associated with defects in stress-induced formation of endocytic vesicles and intracellular MVBs. This suggests LIP5 has a critical role of pathogen/stress responsive MVB biogenesis in broad plant stress responses. However, there has been no direct genetic evidence for the role of MVBs in affecting ABA signaling as mutations of genes essential for MVB biogenesis are often lethal (Haas et al., 2007; Spitzer et al., 2009). In this study, the lip5 mutants decreased sensitivity to $\mathrm{ABA}$ and reduced drought tolerance (Figures 3-5). Using the FM1-43 staining, we further observed that stress- or ABA-induced endocytic activities were increased in wild-type plants but not in the lip5 mutant plants (Figure 6, Supplementary Figure S2). This suggested that LIP5 is necessary for stress-induced vesicle trafficking. We further demonstrated that LIP5 over-expression plants were more sensitive than the 
A

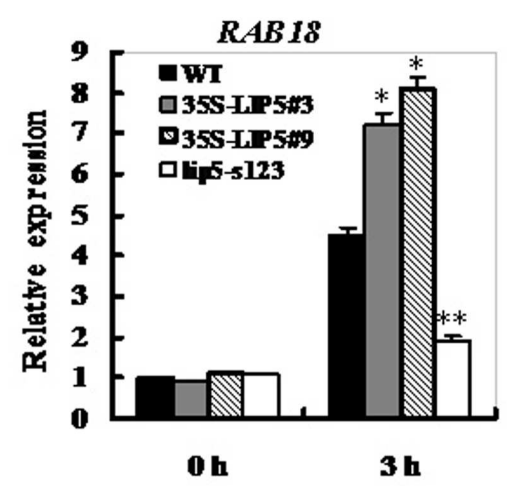

D

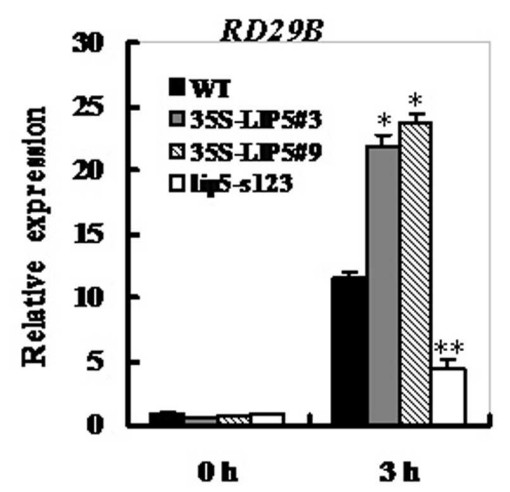

B

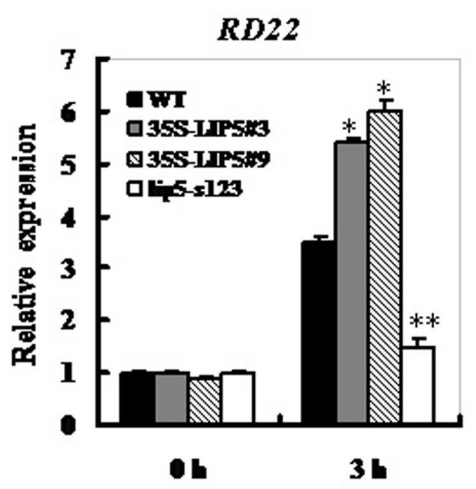

E

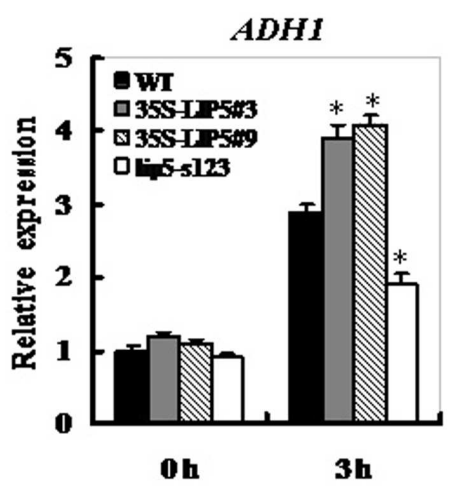

C

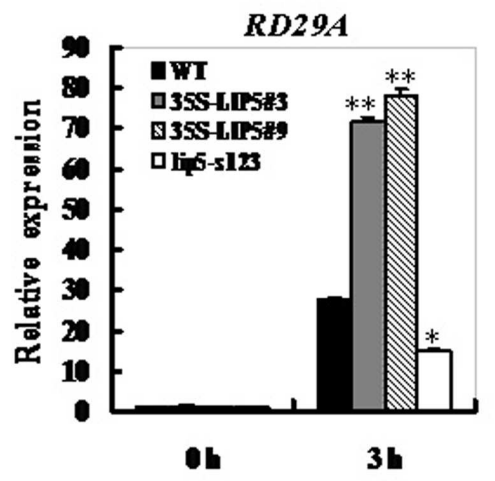

$\mathbf{F}$

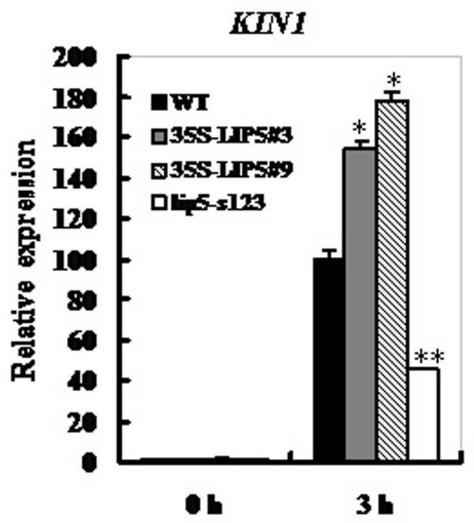

FIGURE 7 | Expression of ABA- and stress-responsive genes in the lip5 and overexpression lines in response to ABA. Three-weeks-old plants were treated with $50 \mu \mathrm{M}$ ABA for 0 and $3 \mathrm{~h}$, and expression of ABA- and stress-responsive genes $R A B 18$ (A), RD22 (B), RD29A (C), RD29B (D), ADH1 (E), and KIN1 (F) was assayed by qRT-PCR in seedlings of WT, lip5-s123, and two WT/35S-LIP5 lines (\#3 and \#9). For each experiment, three technical replicates were conducted. Data shown are mean \pm SE of three independent experiments. $T$-test, with ${ }^{*} P<0.01$; $t$-test, with ${ }^{*} P<0.05$.

wild-type in ABA-induced stomatal closure, whereas the lip5 mutant plants were less sensitive (Figure 5); indicating that LIP5 may act in regulating ABA-mediated stomatal closure. Noticeably, it has been reported that ABA triggers endocytosis of $\mathrm{K}^{+}$channel proteins at the plasma membrane in plant guard cells to control ion transport and transpiration under water stress (Sutter et al., 2007). Based on this report and our results, we speculate that LIP5-regulated endocytosis might be involved in ABA-induced stomatal closure under drought conditions.

In correspondence with these ABA- or drought tolerancerelated phenotypes, expression of several drought- or ABAresponsive marker genes, including $R A B 18, R D 22, R D 29 A$, $R D 29 B, A D H 1$, and KIN1 was significantly down-regulated upon ABA exposure in the lip5 mutant seedlings (Figure 7). RD29A is a drought- and ABA-inducible gene and its expression in the lip5 mutant could be due to the reduction of the upstream bZIP family transcription factors (Shinozaki and YamaguchiShinozaki, 1997). However, it is not sure which of the bZIP factors affected $R D 29 A$ expression. The expression of $R A B 18$ was significantly reduced in the lip5 mutant, similar to that of RD22 (Figures 7A,B). Several MYC and MYB binding elements have been found in the promoter regions of RAB18 and RD22, indicating that both genes may be regulated by the two classes of transcription factors MYB and MYC (Zhang et al., 2007). In the lip5 mutant, reduced expression of LIP5 attenuated ABA signal transduction, which might result in decreased expression of MYC and MYB and finally affect the expression of $R A B 18$ and RD22. Based on previous and our results, it is reasonable to speculate that under drought conditions, LIP5 promotes cell endocytosis and vesicle trafficking, which might accelerate trafficking and sorting of some regulatory proteins such as bZIP, MYC or MYB, and finally results in up-regulation of some ABA-induced downstream genes (such as $R A B 18, R D 22, R D 29 A$, et al.) and ABA response. Future work will be needed to dissect the mechanisms in detail by which the LIP5-regulated MVB biogenesis is involved in ABA signal transduction using the isolated lip5 mutants and LIP5overexpression lines.

\section{AUTHOR CONTRIBUTIONS}

Zongliang Xia designed the research. YH, YW, QC, Ziwei Xu, $\mathrm{WZ}$, and Zongliang Xia performed research and conducted data analyses. Zongliang Xia wrote the manuscript. 


\section{ACKNOWLEDGMENTS}

This work was financially supported by the science and technology R\&D project of Henan (grant no. HYKJ201202). We thank the Arabidopsis Biological Resource Center for providing T-DNA insertion mutant seeds.

\section{SUPPLEMENTARY MATERIAL}

The Supplementary Material for this article can be found online at: http://journal.frontiersin.org/article/10.3389/fpls.2016.00758

FIGURE S1 | Molecular identification of Arabidopsis lip5 mutants.

(A) Structure of the LIP5 locus with the T-DNA insertion site in lip5-s123 and

\section{REFERENCES}

Abe, H., Urao, T., Ito, T., Seki, M., Shinozaki, K., and Yamaguchi-Shinozaki, K. (2003). Arabidopsis AtMYC2 (bHLH) and AtMYB2 (MYB) function as transcriptional activators in abscisic acid signaling. Plant Cell 15, 63-78. doi: $10.1105 /$ tpc.006130

Azmi, I., Davies, B., Dimaano, C., Payne, J., Eckert, D., Babstet, M., et al. (2006). Recycling of ESCRTs by the AAA-ATPase Vps4 is regulated by a conserved VSL region in Vta1. J. Cell Biol. 172, 705-717. doi: 10.1083/jcb.200508166

Babst, M., Wendland, B., Estepa, E. J., and Emr, S. D. (1998). The Vps4p AAA ATPase regulates membrane association of a Vps protein complex required for normal endosome function. EMBO J. 17, 2982-2993. doi: 10.1093/emboj/17.11.2982

Bolte, S., Talbot, C., Boutte, Y., Catrice, O., Read, N. D., and SatiatJeunemaitre, B. (2004). FM-dyes as experimental probes for dissecting vesicle trafficking in living plant cells. J. Microsc. 214, 159-173. doi: 10.1111/j.00222720.2004.01348.x

Bu, Q., Li, H., Zhao, Q., Jiang, H., Zhai, Q., Zhang, J., et al. (2009). The Arabidopsis RING finger E3 ligase RHA2a is a novel positive regulator of abscisic acid signaling during seed germination and early seedling development. Plant Physiol. 150, 463-481. doi: 10.1104/pp.109.135269

Christmann, A., Moes, D., Himmelbach, A., Yang, Y., Tang, Y., and Grill, E. (2006). Integration of abscisic acid signalling into plant responses. Plant Biol. (Stuttg.) 8, 314-325. doi: 10.1055/s-2006-924120

Clough, S. J., and Bent, A. F. (1998). Floral dip: a simplified method for Agrobaterium -mediated transformation of Arabidopsis thaliana. Plant J. 16, 735-743. doi: 10.1046/j.1365-313x.1998.00343.x

Contento, A. L., and Bassham, D. C. (2012). Structure and function of endosomes in plant cells. J. Cell Sci. 125, 3511-3518. doi: 10.1242/jcs.093559

Finkelstein, R. R., Gampala, S. S., and Rock, C. D. (2002). Abscisic acid signaling in seeds and seedlings. Plant Cell (Suppl.) 14, S15-S45.

Fujita, H., Umezuki, Y., Imamura, K., Ishikawa, D., Uchimura, S., Nara, A., et al. (2004). Mammalian class E Vps proteins, SBP1 and mVps2/CHMP2A, interact with and regulate the function of an AAA-ATPase SKD1/Vps4B. J. Cell Sci. 117, 2997-3009. doi: $10.1242 /$ jcs. 01170

Haas, T. J., Sliwinski, M. K., Martinez, D. E., Preuss, M., Ebine, K., Ueda, T., et al. (2007). The Arabidopsis AAA ATPase SKD1 is involved in multivesicular endosome function and interacts with its positive regulator LYST-INTERACTING PROTEIN5. Plant Cell 19, 1295-1312. doi: $10.1105 /$ tpc. 106.049346

Hasegawa, M., Bressan, R., and Pardo, J. M. (2000). The dawn of plant salt tolerance genetics. Trends Plant Sci. 5, 317-319. doi: 10.1016/S1360-1385(00) 01692-7

Jefferson, R. A., Kavanagh, T. A., and Bevan, M. W. (1987). GUS fusions: betaglucuronidase as a sensitive and versatile gene fusion marker in higher plants. EMBO J. 6, 3901-3907.

Kawasaki, S., Borchert, C., Deyholos, M., Wang, H., Brazille, S., Kawai, K., et al. (2001). Gene expression profiles during the initial phase of salt stress in rice. Plant Cell 13, 889-905. doi: 10.1105/tpc.13.4.889 lip5-s145 mutants. The insertion site is marked by a white arrow, exons are indicated as white boxes, and untranslated regions by black boxes. P1, forward primer; P2, reverse primer; LBb1, primer specific to the T-DNA left border. (B) Diagnostic PCR of the T-DNA inserted in two different loci of LIP5. DNA from homozygous insertion lines of lip5-s123 and lip5-s145 were used. M, molecular mass markers. Primers used for PCR are indicated above each lane.

FIGURE S2 | Phenotypes of LIP5 mutant and wild type plants in ABA-induced endocytosis in leaves. Two weeks-old lip5-s123 and WT plants were treated by spraying $50 \mu \mathrm{M}$ ABA, and after $1 \mathrm{~h}, \mathrm{ABA}$-induced endocytosis was analyzed from control and treated Arabidopsis leaf epidermis using FM1-43 staining. (A) Representative confocal images of Arabidopsis guard cells of WT and lip5 mutant plants after FM1-43 staining. Bar $=10 \mu \mathrm{m}$. (B) Signal intensity of internalized FM1-43 in Arabidopsis leaf guard cells of WT and lip5 mutant plants. Means and SE were calculated from images of 10 independent leaves for each genotype. $T$-test, with ${ }^{* *} P<0.01$. In both $(\mathbf{A}, \mathbf{B})$ experiments were repeated two times with similar results.

Kermode, A. R. (2005). Role of abscisic acid in seed dormancy. J. Plant Growth Regul. 24, 319-344. doi: 10.1007/s00344-005-0110-2

Knight, H., and Knight, M. R. (2001). Abiotic stress signalling pathways: specificity and cross-talk. Trends Plant Sci. 6, 262-267. doi: 10.1016/S13601385(01)01946-X

Kurkela, S., and Borg-Franck, M. (1992). Structure and expression of kin2, one of two cold- and ABA-induced genes of Arabidopsis thaliana. Plant Mol. Biol. 19, 689-692. doi: 10.1007/BF00026794

Lang, V., and Palva, E. T. (1992). The expression of a rab-related gene, rab18, is induced by abscisic acid during the cold acclimation process of Arabidopsis thaliana (L.) Heynh. Plant Mol. Biol. 20, 951-962. doi: 10.1007/BF00027165

Lescot, M., Dehais, P., Thijs, G., Marchal, K., Moreau, Y., Van de Peer, Y., et al. (2002). PlantCARE, a database of plant cis-acting regulatory elements and a portal to tools for in silico analysis of promoter sequences. Nucleic. Acids Res. 30, 325-327. doi: 10.1093/nar/30.1.325

Leung, J., and Giraudat, J. (1998). Abscisic acid signal transduction. Annu. Rev. Plant Physiol. Plant Mol. Biol. 49, 199-222. doi: 10.1146/annurev.arplant.49.1.199

Lim, C. W., and Lee, S. C. (2014). Functional roles of the pepper MLO protein gene, CaMLO2, in abscisic acid signaling and drought sensitivity. Plant Mol. Biol. 85, 1-10. doi: 10.1007/s11103-013-0155-8

Livak, K. J., and Schmittgen, T. D. (2001). Analysis of relative gene expression data using real-time quantitative PCR and the 2(-Delta Delta C (T)) method. Methods 25, 402-408. doi: 10.1006/meth.2001.1262

Lottridge, J. M., Flannery, A. R., Vincelli, J. L., and Stevens, T. H. (2006). Vtalp and Vps46p regulate the membrane association and ATPase activity of Vps4p at the yeast multivesicular body. Proc. Natl. Acad. Sci. U.S.A. 103, 6202-6207. doi: 10.1073/pnas.0601712103

Nakashima, K., Fujita, Y., Katsura, K., Maruyama, K., Narusaka, Y., Seki, M., et al. (2006). Transcriptional regulation of ABI3- and ABA- responsive genes including RD29B and RD29A in seeds, germinating embryos, and seedlings of Arabidopsis. Plant Mol. Biol. 60, 51-68. doi: 10.1007/s11103-005-2418-5

Reyes, F. C., Buono, R., and Otegui, M. S. (2011). Plant endosomal trafficking pathways. Curr. Opin. Plant Biol. 14, 666-673. doi: 10.1016/j.pbi.2011. 07.009

Scott, A., Chung, H. Y., Gonciarz-Swiatek, M., Hill, G. C., Whitby, F. G., Gaspar, J., et al. (2005). Structural and mechanistic studies of VPS4 proteins. EMBO J. 24, 3658-3669. doi: 10.1038/sj.emboj.7600818

Shahriari, M., Keshavaiah, C., Scheuring, D., Sabovljevic, A., Pimpl, P., Hausler, R. E., et al. (2010). The AAA-ATPase AtSKD1 contributes to vacuolar maintenance of A. thaliana. Plant J. 64, 71-85. doi: 10.1111/j.1365313X.2010.04310.x

Shiflett, S. L., Ward, D. M., Huynh, D., Vaughn, M. B., Simmons, J. C., and Kaplan, J. (2004). Characterization of Vtalp, a class E Vps protein in Saccharomyces cerevisiae. J. Biol. Chem. 279, 10982-10990. doi: 10.1074/jbc.M312669200

Shinozaki, K., and Yamaguchi-Shinozaki, K. (1997). Gene expression and signal transduction in water-stress response. Plant Physiol. 115, 327-334. doi: $10.1104 /$ pp.115.2.327 
Spitzer, C., Reyes, F. C., Buono, R., Sliwinski, M. K., Haas, T. J., and Otegui, M. S. (2009). The ESCRT-related CHMP1A and B proteins mediate multivesicular body sorting of auxin carriers in Arabidopsis and are required for plant development. Plant Cell 21, 749-766. doi: 10.1105/tpc.108.064865

Sutter, J. U., Sieben, C., Hartel, A., Eisenach, C., Thiel, G., and Blatt, M. R. (2007). Abscisic acid triggers the endocytosis of the Arabidopsis KAT1 K+ channel and its recycling to the plasma membrane. Curr. Biol. 17, 1396-1402. doi: 10.1016/j.cub.2007.07.020

Vild, C. J., Li, Y., Guo, E. Z., Liu, Y., and Xu, Z. (2015). A novel mechanism of regulating the ATPase VPS4 by its cofactor LIP5 and the endosomal sorting complex required for transport (ESCRT)- III protein CHMP5. J. Biol. Chem. 290, 7291-7303. doi: 10.1074/jbc.M114.616730

Wang, F., Shang, Y., Fan, B., Yu, J. Q., and Chen, Z. (2014). Arabidopsis LIP5, a positive regulator of multivesicular body biogenesis, is a critical target of pathogen-responsive MAPK cascade in plant basal defense. PLoS Pathog. 10:e1004243. doi: 10.1371/journal.ppat.1004243

Wang, F., Yang, Y., Wang, Z., Zhou, J., Fan, B., and Chen, Z. (2015). A critical role of Lyst-Interacting protein 5 , a positive regulator of multivesicular body biogenesis, in plant responses to heat and salt stresses. Plant Physiol. 169, 497-511. doi: 10.1104/pp.15.00518

Ward, D. M. V., Vaughn, M. B., Shiflett, S. L., White, P. L., Pollock, A. L., Hill, J., et al. (2005). The role of LIP5 and CHMP5 in multivesicular body formation and HIV-1 budding in mammalian cells. J. Biol. Chem. 280, 10548-10555. doi: 10.1074/jbc.M413734200

Winter, V., and Hauser, M. T. (2006). Exploring the ESCRTing machinery in eukaryotes. Trends Plant Sci. 11, 115-123. doi: 10.1016/j.tplants.2006. 01.008

Xia, Z., Su, X., Liu, J., and Wang, M. (2013). The RING-H2 finger gene 1 (RHF1) encodes an E3 ubiquitin ligase and participates in drought stress response in Nicotiana tabacum. Genetica 141, 11-21. doi: 10.1007/s10709-013-9702-0

Xia, Z., Sun, K., Wang, M., Wu, K., and Zhang, H. (2012). Overexpression of a maize sulfite oxidase gene in tobacco enhances tolerance to sulfite stress via sulfite oxidation and CAT-mediated $\mathrm{H}_{2} \mathrm{O}_{2}$ scavenging. PLoS ONE 7:e37383. doi: 10.1371/journal.pone.0037383

Xia, Z., Zhang, X., Li, J., Su, X., and Liu, J. (2014). Overexpression of a tobacco J-domain protein enhances drought tolerance in transgenic Arabidopsis. Plant Physiol. Biochem. 83, 100-106. doi: 10.1016/j.plaphy.2014.07.023

Xiong, L., Schumaker, K. S., and Zhu, J. K. (2002). Cell signaling during cold, drought, and salt stress. Plant Cell 14(Suppl.), S165-S183.

Yamaguchi-Shinozaki, K., and Shinozaki, K. (2006). Transcriptional regulatory networks in cellular responses and tolerance to dehydration and cold stresses. Annu. Rev. Plant Biol. 57, 781-803. doi: 10.1146/annurev.arplant.57.032905.105444

Yeo, S. C., Xu, L., Ren, J., Boulton, V. J., Wagle, M. D., Liu, C., et al. (2003). Vps20p and Vtalp interact with Vps4p and function in multivesicular body sorting and endosomal transport in Saccharomyces cerevisiae. J. Cell Sci. 116, 3957-3970. doi: $10.1242 /$ jcs.00751

Zhang, Y. Y., Yang, C. W., Li, Y., Zheng, N. Y., Chen, H., Zhao, Q. Z., et al. (2007). SDIR1 is a RING finger E3 ligase that positively regulates stressresponsive abscisic acid signaling in Arabidopsis. Plant Cell 19, 1912-1929. doi: $10.1105 /$ tpc. 106.048488

Zhu, J. K. (2002). Salt and drought stress signal transduction in plants. Annu. Rev. Plant Biol. 53, 247-273. doi: 10.1146/annurev.arplant.53.091401.143329

Conflict of Interest Statement: The authors declare that the research was conducted in the absence of any commercial or financial relationships that could be construed as a potential conflict of interest.

Copyright (c) 2016 Xia, Huo, Wei, Chen, Xu and Zhang. This is an open-access article distributed under the terms of the Creative Commons Attribution License (CC BY). The use, distribution or reproduction in other forums is permitted, provided the original author(s) or licensor are credited and that the original publication in this journal is cited, in accordance with accepted academic practice. No use, distribution or reproduction is permitted which does not comply with these terms. 